\title{
Two-dimensional Mapping of Bulk Residual Stress Using Cut Mouth Opening Displacement
}

\author{
C. R. Chighizola ${ }^{1}$ M. R. Hill ${ }^{1}$ (i)
}

Received: 25 November 2020 / Accepted: 8 June 2021 / Published online: 17 August 2021

(c) The Author(s) 2021

\begin{abstract}
Background Prior work described an approach for mapping the two-dimensional spatial distribution of biaxial residual stress in plate-like samples, the approach combining multiple slitting measurements with elastic stress analysis.

Objective This paper extends the prior work by applying a new variation of the slitting method that uses measurements of cut mouth opening displacement (CMOD) rather than back-face strain (BFS).

Methods First, CMOD slitting is validated using an experiment where: BFS and CMOD are measured simultaneously on the same sample during incremental slitting; two residual stress profiles are computed, one from the BFS data and a second from the CMOD data; and the two residual stress profiles are compared. Following validation, multiple adjacent CMOD slitting measurements are used to construct two-dimensional maps of residual stress in plates cut from quenched aluminum. Results The two residual stress versus depth profiles, each computed separately from BFS or CMOD data, are in agreement, with compression near the plate boundaries (-150 MPa) and tension near the plate center (100 MPa); differences between the two stress profiles have a maximum of $25 \mathrm{MPa}$ and a RMS of 7.2 MPa. Repeated biaxial residual stress mapping measurements show the CMOD technique is repeatable, and complementary contour method measurements show the mappings are valid. Aspects of CMOD and BFS deformations during slitting are also described and show they are generally complementary but that CMOD slitting is favorable in narrow samples.
\end{abstract}

Keywords Residual stress measurement $\cdot$ Slitting method $\cdot$ Cut mouth opening displacement

\section{Introduction}

Quenching, the rapid cooling of a hot workpiece, is a mainstay of material processing in a variety of metallic alloys [1]. A drawback of quenching is the introduction of bulk residual stress (BRS) which, if not well understood, can lead to unexpected machining distortion during manufacturing and to performance issues during service [2]. Engineering models can help alleviate manufacturing and service issues. Formulation and validation of engineering models require measurements of BRS in quenched parts [3]. BRS fields in typical parts can be measured using methods based on diffraction or mechanical relaxation [4] with the mechanical relaxation being easier to apply for bulk stress fields in a

M. R. Hill

mrhill@ucdavis.edu

1 Department of Mechanical and Aerospace Engineering, University of California, One Shields Avenue, Davis, CA 95616, United States typical engineering laboratory. The slitting method is useful for two-dimensional stress fields in particular, being relatively simple and offering good precision.

This work describes experimental measurements of two in-plane components of the residual stress tensor field in plate-shaped aluminum parts that had been cut perpendicular to the length of long rectangular quenched bars. Given the uniformity of quench far from the ends of a quenched bar, the residual stress fields in the plates are assumed to be twodimensional, varying only with in-plane position and independent of position into the plate thickness. Measurements are made using the slitting method, which is a mechanical relaxation technique for measuring one component of residual stress as a function of position along a line in a prismatic body [5]. The slitting method was pioneered by Finnie and co-workers [5, 6]. In a typical slitting experiment, a cut is made across the width of a sample in increments of depth using wire electric discharge machining (EDM). A strain gage located at the opposite end of the sample measures deformation at each cut depth, which occurs due to 
the release of stress on the cut plane. The residual stress as a function of position along the cut is calculated from strain versus cut depth data by solving an inverse problem, as described by Schajer and Prime [7]. Prior work shows that the slitting method is repeatable and applicable to measuring BRS in quenched aluminum [8].

Work done by Olson and Hill showed that the slitting method is sufficient to measure and resolve a two-dimensional (2D) map of residual stress using multiple measurements [9]. The 2D mapping of BRS as a function of position requires slitting measurements on several planes, where neighboring measurements are spaced apart to provide a map of BRS with sufficient resolution. In theory, successive measurements could be made far enough apart to not be affected by prior measurements, but at the cost of map resolution. Since quenched parts have strong stress gradients closer spacing is more suitable to achieve a BRS map of sufficient resolution. A closer spacing requires accounting for the effect of prior measurements on subsequent measurements by use of a stress correction, as described in earlier work [10, 11]. Assuming elastic behavior, superposition is used to combine the measured stress and stress corrections to resolve a $2 \mathrm{D}$ map of BRS [9].

Performing multiple measurements on several parts using the typical slitting approach can be expensive and timeconsuming. Here we develop and apply a new variation of the slitting method that uses cut mouth opening displacement (CMOD), rather than the typical back face strain (BFS), and we apply this technique to map two components of BRS in plate-shaped aluminum parts as a function of in-plane position. CMOD measured using a typical clip-style gage (such as used in fracture mechanics testing [12]) has the practical advantage of not requiring application of a strain gage with adhesive and associated waterproofing. The first objective of this work is to validate CMOD slitting by performing an experiment that compares residual stress measured using CMOD and BFS simultaneously. The second objective is to apply CMOD slitting to map two components of BRS in quenched plates. The third objective is to validate the CMOD slitting measurements by making contour method [13] measurements in identically prepared plates and comparing the results from CMOD slitting and contour.

\section{Methods}

\section{Material and Sample Description}

The material used in this study is AA 7050-T7451, a common aerospace grade alloy used in airframe structures. The temper designation T7451 indicates a rolled plate artificially over-aged and stretched for stress relief. The stress relieved AA 7050-T7451 was purchased as a square plate, $1250 \mathrm{~mm}$
(L) by $1250 \mathrm{~mm}$ (LT) and $102 \mathrm{~mm}$ (ST) thick (see Fig. 1). The plate was cut into 6 blocks, each $66 \mathrm{~mm}$ (LT) by 206 $\mathrm{mm}$ (L) by $102 \mathrm{~mm}$ (ST). Three of these blocks, D, F, and $\mathrm{G}$ were heat treated to $\mathrm{T} 74$ condition, which produces high residual stress. The heat treatment follows industry specification [14] where the plate is heated to $475{ }^{\circ} \mathrm{C}$ quenched to room temperature then aged in two stages at $107^{\circ} \mathrm{C}$ for 6 to 8 $\mathrm{h}$ and the $163{ }^{\circ} \mathrm{C}$ for 24 to $30 \mathrm{~h}$. After heat treatment the ends of the quenched blocks were discarded, and 15 individual plate-shaped samples were cut from the material remaining in each block. A labeling scheme ties each sample to its location, as shown in Fig. 1. The plate geometry is 206 $\mathrm{mm}$ long $(H)$ by $102 \mathrm{~mm}$ wide $(W)$, and $28 \mathrm{~mm}$ thick $(t)$. A coordinate frame is defined relative to the dimensions, with $x$ along $H, y$ along $W$, and $z$ along $t$. Because of the spatial uniformity of quenching, and discarding of the ends of the quenched plates, we expect that all samples have nearly identical residual stress fields.

\section{Validate CMOD Versus BFS Slitting}

As a first step, incremental slitting is performed while BFS and CMOD are measured simultaneously. Given that there are no physical or electrical connections between the BFS and CMOD measurement equipment, the two measurements are assumed independent. The experiment configuration is shown in Fig. 2, where the slit is cut along the positive $y$ direction at the mid-width and the distance from the slit to the plate edge is $s=H / 2$. A CMOD gage is attached at the cut mouth $(y=0)$ and a strain gage at the back face $(y=W)$. CMOD is measured using a commercial clip-type gage (MTS 632.03E-30) with initial gage length $L_{\mathrm{M}}=6.35 \mathrm{~mm}(0.062 \mathrm{~W})$. BFS is measured using a metallic foil gage with active length $L_{\varepsilon}=1.575 \mathrm{~mm}(0.015 \mathrm{~W})$. Two notches $6.35 \mathrm{~mm}$ apart and $1.0 \mathrm{~mm}$ deep are cut at the front

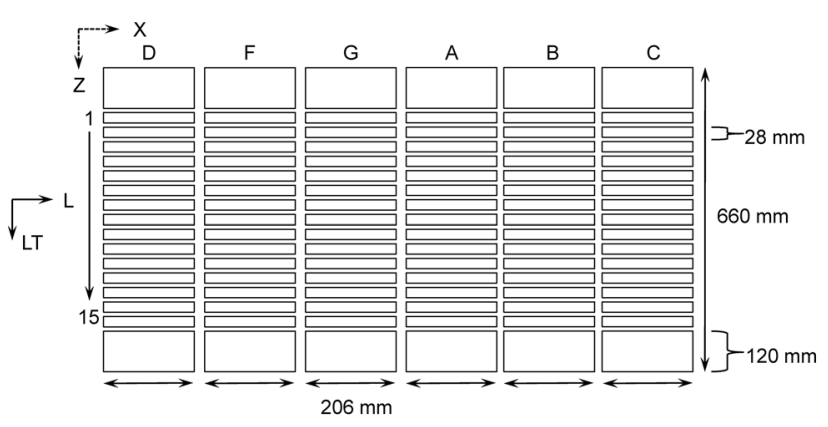

Fig. 1 Diagrams of the sample labeling scheme and locations of samples where the longitudinal rolling direction (L) is along $\mathrm{x}$ and the long transverse direction (LT) is along z. Blocks D, F, and G had additional heat treatment. Individual samples are coded as \%\#, where $\%$ is $\mathrm{A}, \mathrm{B}, \mathrm{C}, \mathrm{D}, \mathrm{F}$, or $\mathrm{G}$, indicating the parent block, and \# indicates the position of the sample in the section (positions 1 to 15 shown at left). The $120 \mathrm{~mm}$ blocks are discarded to remove end effects 


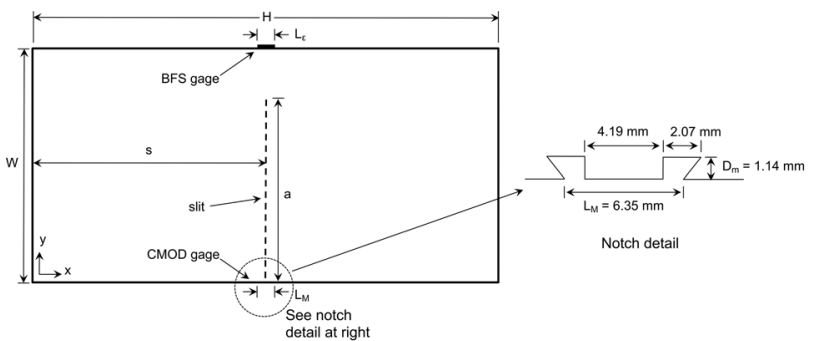

Fig. 2 Measurement geometry for the slitting experiments with CMOD notch detail at right: $\mathrm{W}$ is the part width, $\mathrm{H}$ is the part length, $s$ is the distance from the edge to the slit plane, $L_{\varepsilon}$ is the strain gage length, and $\mathrm{L}_{\mathrm{M}}$ is the CMOD gage length

of the plate to attach the CMOD gage prior to cutting the slit (detail in Fig. 2). Measurements of BFS and CMOD are made independently as a slit is cut to 40 equal increments of depth, a typical number of cuts (e.g., [5]), with cut depths $a=2.54,5.08, \ldots, 97.8 \mathrm{~mm}$. Cut closure is avoided following procedures used by Olson and Hill [9], which comprise a visual inspection at each cut depth and cutting back to the slit mouth to maintain a gap between cut faces when needed (a gap between cut planes avoids mechanical cut face contacts that would invalidate the stress calculation scheme [9]). Following the experiment, residual stress is computed from deformation (BFS or CMOD) versus cut depth data using the approach described by Schajer and Prime [7]. Compliance matrices needed for the stress computation are developed using finite element modeling as described in earlier work (see $[5,15]$ ), where key features include a refined mesh (400 uniform elements adjacent to the crack line), tractions applied to a slit of zero width, and use of actual dimensions and locations of the gages measuring BFS and CMOD).

A correction step is performed for CMOD slitting to account for the stress released when cutting the notch used to accommodate the CMOD gage. An approximate correction for the effect of stress released due to cutting the notch is developed using a stress analysis for idealized initial stress fields representative of expected residual stresses. The stress analysis uses the finite element method (FEM) and reflects a 2D domain with and without a notch; the analysis is repeated for piecewise constant, piecewise linear, and sinusoidal stress profiles of unit magnitude, as shown in Fig. 3. Each stress field is balanced, having compressive stress near the edges and tensile stress in the interior, typical of residual stress in quenched parts. The representative stress profiles are imposed as an initial condition in models with either a notched or unnotched geometry. Residual stress at the slit plane is calculated. The difference in stress between notched and unnotched geometries is a new profile $e(y)$, referred to as the notch error, representing the stress released during the notching process
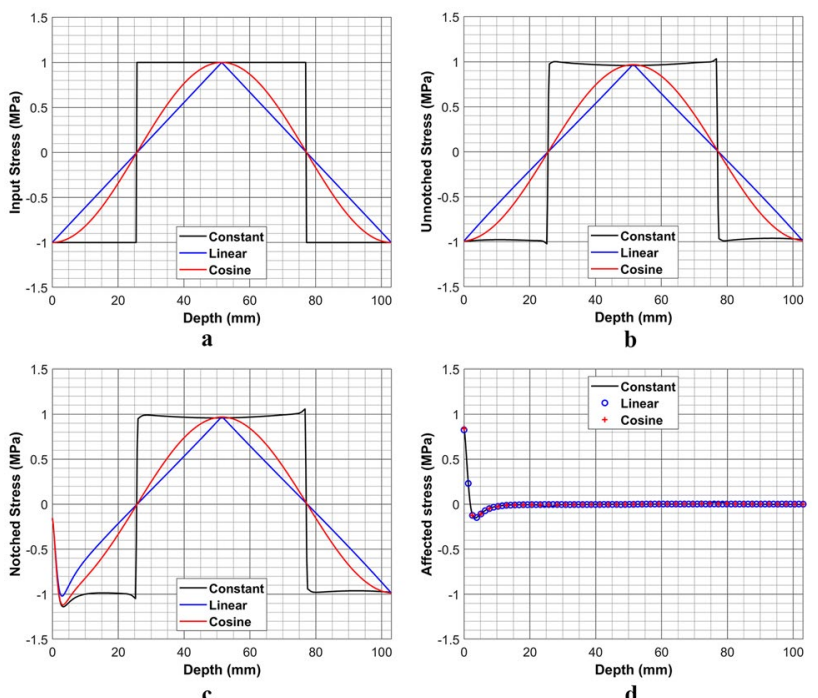

Fig. 3 Plots of (a) representative model input stresses, (b) extracted stress profiles from an unnotched model, (c) extracted stress profiles from a notch model, and (d) the notch error (notched stress unnotched stress) profiles at the slit plane

$e(y)=\sigma_{x x, \text { notched }}(y)-\sigma_{x x, \text { unnotched }}(y)$

All three representative stress profiles exhibit very nearly the same notch error $e(y)$ (see Fig. 3(d)), and $e(y)$ from the sinusoidal stress profile was taken as a useful estimate of the notch error. A scaling factor, $\alpha$, is used to scale $e(y)$ for a particular measurement. For a given measurement, $\alpha$ is computed from the measured (post-notch) stress as the average of the measured stress over the distance $0<x<10 \mathrm{~mm}$. Combining the notch affected stress, the scale factor, and the error corrects for the stress released due to cutting the notch

$\sigma_{x x, \text { total }}=\sigma_{x x, \text { measured }}+\alpha e(y)$

Figure 4 shows an example of a post-notch stress correction using a sinusoidal stress profile as the idealized stress field.
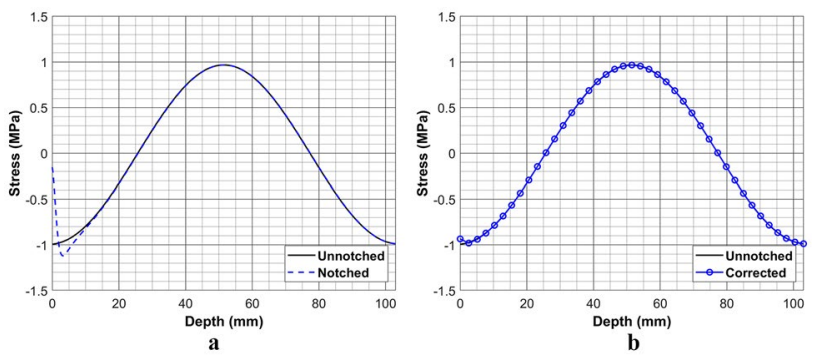

Fig. 4 Plots representing the notch correction process for a model with aspect ratio $H=2 W$ with (a) the unnotched and notched profile from a representative cosine stress input, and (b) a corrected and unnotched profile 


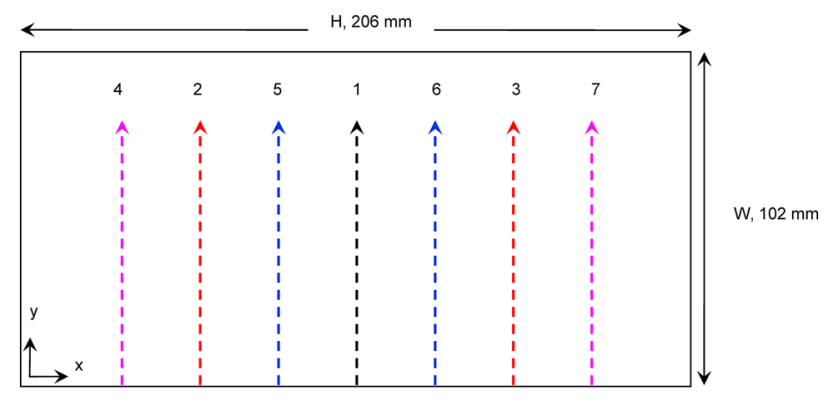

Fig. 5 Bisection scheme for longitudinal bulk residual stress $\left(\sigma_{\mathrm{xx}}\right)$ measurements; numeric labels reflect the sequence of measurements

\section{Bi-axial Mapping}

The two normal in-plane components of BRS are measured on several planes using CMOD slitting to create two-dimensional maps of the BRS in the plates. Following the earlier work of Olson and Hill [9], we employ a bisecting scheme to ensure measurements are insensitive to shear stress error. To map $\sigma_{x x}(x, y)$, a first measurement is made at $x=H / 2$ cutting in the positive y-direction. This produces a profile of $\sigma_{x x}(H / 2$, $y$ ). Successive slitting measurements are made at $x=H / 4$ and $3 H / 4$, then $H / 8$ and $3 H / 8$, and finally $5 H / 8$ and $7 H / 8$, for a total of 7 measurements in each plate, as shown in Fig. 5. The number of measurements (7) was selected based on anticipated stress gradients and reasonable level of effort; additional measurements may be beneficial. Measured stresses are combined to produce a map of $\sigma_{x x}(x, y)$ with the aid of linear interpolation and traction-free boundary conditions (i.e., $\sigma_{x x}(0, y)$ $=0$ and $\left.\sigma_{x x}(H, y)=0\right)$. To map $\sigma_{y y}(x, y)$, measurements are made at several $y$ locations. An initial slitting measurement of $\sigma_{x x}(y)$ is made at $x=H / 2$ to reduce the 102 by $206 \mathrm{~mm}$ plate into two 102 by $103 \mathrm{~mm}$ pieces. Subsequent measurements of $\sigma_{y y}(x)$ are made at $y=W / 2, W / 4$, and $3 W / 4$ in the two halves, where for the left half, cuts progress along positive $x$, and for the right half, cuts progress along negative $x$. On one sample an additional measurement was performed at $y=W / 8$ to assess $\sigma_{y y}(x)$ closer to the sample edge. Measured stresses are combined to produce a map of $\sigma_{y y}(x, y)$ with the aid of linear interpolation and traction-free boundary conditions (i.e., $\sigma_{y y}(x$, $0)=0$ and $\left.\sigma_{y y}(x, W)=0\right)$. A diagram depicting the bisecting scheme used to map $\sigma_{y y}(x, y)$ is shown in Fig. 6 .

When mapping residual stress using adjacent cuts, the effect of a prior measurement on a subsequent measurement must be accounted for using a stress correction because a prior measurement releases stress in adjacent material. The stress correction approach follows the procedure described in [9] that relies on elastic superposition and is based on the earlier work of Pagliaro and Prime [11]. In a first step, stress determined in the prior measurement is used as a traction boundary condition in a 2D FEM stress analysis whose geometry reflects the sample configuration at the end of the prior measurement. In

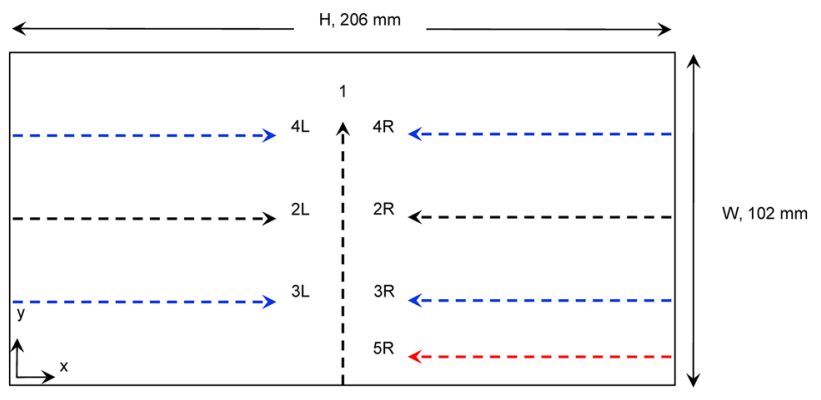

Fig. 6 Bisection scheme for transverse bulk residual stress $\left(\sigma_{\mathrm{yy}}\right)$ measurements; numeric labels reflect the sequence of measurements

a second step, stress determined in the analysis at the location of a subsequent measurement is recorded and comprises the stress correction. In the final step, the stress correction is added to results of the subsequent measurement. The orientation of a subsequent measurement plane can be either parallel to (as [9]) or normal to (as in [10] and [11]) the prior cut plane. The analyses for the stress corrections in this work are simplified by the fact that the sectioning plan includes subsequent measurements in rectangular plates of only 3 different aspect ratios (having width (along the slit) divided by height (perpendicular to the slit) equal to 1,2 , or 4 ).

\section{Validation of Approach with Contour Method Measurements}

The contour method is used in this study to measure BRS in replicate samples and provide validation of the $2 \mathrm{D}$ residual stress fields determined by mapping with CMOD slitting. In a typical contour method experiment, a cut is made on a selected measurement plane using a wire EDM, the cut surface topography is measured, and the topography data are used to compute residual stress [16]. The contour method, like slitting, measures a single component of in-plane normal residual stress as a function of position on a cut plane. However, unlike slitting, the contour method resolves the residual stress field in two dimensions over the cut plane. Two contour measurements are made in this work, one measurement in each of two samples. The first measures $\sigma_{x x}(y, z)$ at $x=H / 2$ and the second measures $\sigma_{y y}(x, z)$ at $y=W / 2$. Samples are cut using wire EDM with finish cut settings [16]. Surface topography is measured using a laser scanning profilometer at a grid of points with $0.2 \mathrm{~mm}$ spacing on the cut surfaces. The stress normal to the cut plane is determined by an elastic FEM analysis using smoothed surface topography as a displacement input field [16]. To compare slitting and contour method results, the through-thickness average stress (average along $z$ at a set of locations along the other coordinate of the contour plane ( $x$ or $y$ ) is computed from the planar contour data and compared to the one-dimensional residual stress profiles determined by slitting. 


\section{Summary of Measurements}

A total of 29 slitting measurements are made on samples D1, F7, F8 and G14 to determine the longitudinal and transverse components of BRS. On samples F8 and G14, measurements of $\sigma_{x x}$ are made at $x=H / 2, H / 4,3 H / 4, H / 8,3 H / 8,5 H / 8$, and $7 H / 8$ (reference Fig. 5). On samples D1 and F7, single measurements of $\sigma_{x x}$ are made at $x=H / 2$, creating two half samples for subsequent measurements of $\sigma_{y y}$ at $y=W / 2, W / 4$, and $3 W / 4$ (reference Fig. 6); one additional measurement of $\sigma_{y y}$ is made at $y=W / 8$ in the right half of sample F7. The initial measurement of $\sigma_{x x}$ at $x=H / 2$ on sample F7 included simultaneous measurement of BFS and CMOD. For validation of the slitting results, single contour measurements are made on samples G7 at $x=H / 2$ and G8 at $y=W / 2$. Measurements are summarized in Table 1.

\section{Results}

\section{CMOD Slitting Validation}

Figure 7 shows data from the slitting measurement having simultaneous CMOD and BFS measurements for $\sigma_{x x}(y)$ on sample F7 at $x=H / 2$. BFS and CMOD versus cut depth are shown in Fig. 7(a) and (b), respectively, and exhibit similar trends but opposite sign. Residual stress computed independently from each source of data are shown in Fig. 7(c). The notch correction of (Equation (2)) was not applied to either residual stress field in Fig. 7(c), so the data reflect stress in the notched plate. The data are in general agreement with a region $(y<15 \mathrm{~mm}(0.074 W))$ where discrepancy is up to $25 \mathrm{MPa}$ but with agreement in other areas $(y>15 \mathrm{~mm})$ to better than $12 \mathrm{MPa}$. The BRS is compressive within $25 \mathrm{~mm}$ of the sample edges with magnitudes between -118 and $-142 \mathrm{MPa}$, and tensile in a $50 \mathrm{~mm}$ band near the sample middle with a peak magnitude around $100 \mathrm{MPa}$. The differences in stress computed from BFS or CMOD for $y<15 \mathrm{~mm}$ may be an effect of the notch used to
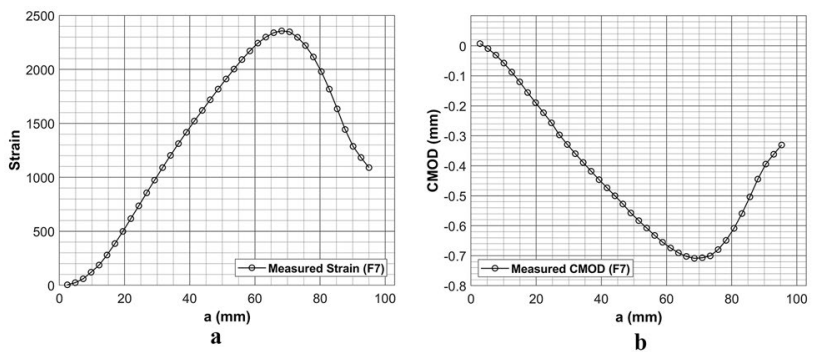

b

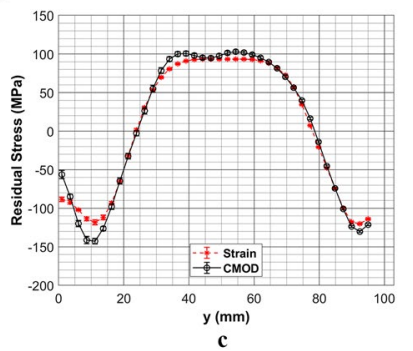

Fig. 7 Plots of (a) measured strain, (b) measured CMOD, and (c) a comparison of longitudinal stress measured using both CMOD and back face strain techniques from a single slitting measurement at $H / 2$ in the y-direction on sample $\mathrm{F} 7$ with $H=2 \mathrm{~W}$

accommodate the CMOD gage, the relatively low sensitivity of the strain gage at small cut depths, or both. The root mean square (RMS) difference over all depths between stress computed from BFS or CMOD is 7.2 MPa. Overall, the agreement (Fig. 7(c)) supports using CMOD slitting for subsequent measurements.

\section{Application of Notch Correction}

Figure 8 shows the notch correction for the measurement of Fig. 7. The correction stress profile, $e(y)$, is from the idealized sinusoidal stress field (see Fig. 3) and a scaling factor calculated from the measured stress. The correction is above $10 \%$ of peak measured stress for $y<5 \mathrm{~mm}$ with the peak level of corrected stress being $38 \mathrm{MPa}$. The notch correction falls below $5 \%$ of peak measured stress for $y>8 \mathrm{~mm}$.

Table. 1 Summary of samples, measured stress components, measurement methods, and locations

\begin{tabular}{|c|c|c|c|}
\hline Sample & Stress Component & Method & Locations \\
\hline \multirow[t]{2}{*}{ F7 } & $\sigma_{x x}$ & CMODBFS & $\begin{array}{l}x=H / 2 \\
x=H / 2\end{array}$ \\
\hline & $\sigma_{y y}$ & CMOD & $\begin{array}{l}y=W / 8 \text { (right half only) } \\
y=W / 4, W / 2, \text { and } 3 W / 4 \text { (both halves) }\end{array}$ \\
\hline \multirow[t]{2}{*}{ D1 } & $\sigma_{x x}$ & CMOD & $x=H / 2$ \\
\hline & $\sigma_{y y}$ & CMOD & $y=W / 4, W / 2$, and $3 W / 4$ (both halves) \\
\hline F8 & $\sigma_{x x}$ & CMOD & $x=H / 8, H / 4,3 H / 8, H / 2,5 H / 8,3 H / 4$, and $7 H / 8$ \\
\hline G14 & $\sigma_{x x}$ & CMOD & $x=H / 8, H / 4,3 H / 8, H / 2,5 H / 8,3 H / 4$, and $7 H / 8$ \\
\hline G8 & $\sigma_{y y}$ & Contour & $\mathrm{y}=W / 2$ \\
\hline G7 & $\sigma_{x x}$ & Contour & $\mathrm{x}=H / 2$ \\
\hline
\end{tabular}




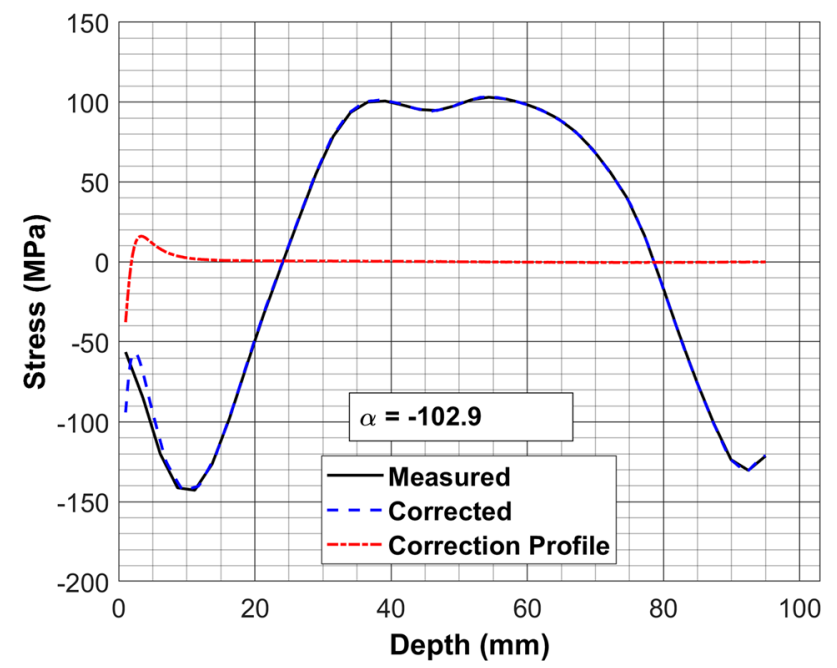

Fig. 8 An application of the correction process to the measurement at $x=H / 2$ on sample $\mathrm{F} 7$

\section{Longitudinal BRS Mapping}

Figure 9 shows results of $\sigma_{x x}$ mapping measurements on multiple planes in sample F8, comprising CMOD versus cut depth, measured stress, stress corrections from prior cuts, and total stress. Compared to CMOD measured for cut 1 , there is a systematic decrease in the magnitude of measured CMOD for subsequent cuts (see Fig. 9(a)). This is consistent with the release of stress in prior measurements. Measured stress data fall into pairs (Fig. 9(b)), with cuts at symmetric positions being similar ( 2 and 3, 4 and 7, 5 and 6). The stress corrections (Fig. 9(c)) systematically decrease

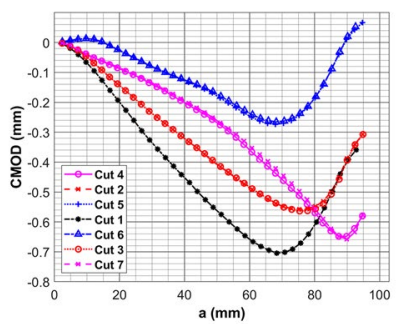

a

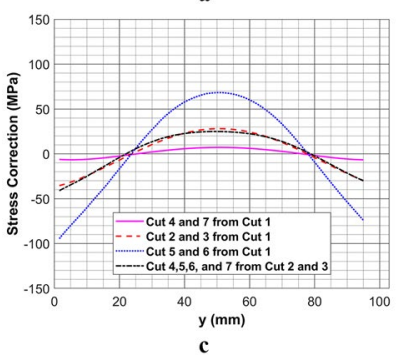

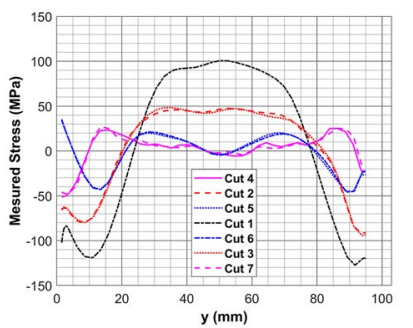

b

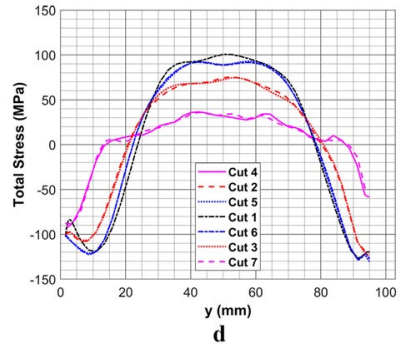

Fig. 9 Data for $\sigma_{x x}$ measurements in sample F8, (a) CMOD versus cut depth, (b) measured stress (c) stress corrections and (d) total stress
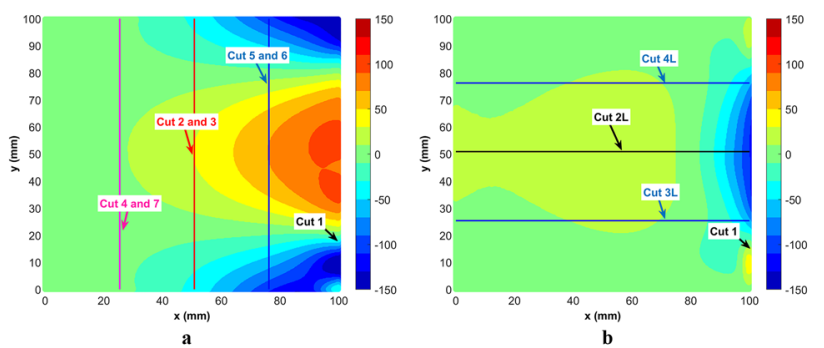

Fig. 10 Color maps of stress corrections from cut 1 on sample F8 for (a) $\sigma_{\mathrm{xx}}$, and (b) $\sigma_{\mathrm{yy}}$ (half of geometry shown; color scale in MPa; lines indicate locations of subsequent measurements)

in magnitude for subsequent measurement locations further from the slit plane with the effect of cut 1 being significant at cut locations 5 and 6 but quite small at locations 4 and 7. Figure 10 shows a stress analysis result for cut 1 in sample F8, having a post-measurement aspect ratio (height to width) of 1 and using stress measured in cut 1 as a traction boundary condition. The color map of Fig. 10(a) shows the $\sigma_{x x}$ correction at different locations, which is identical for planes on either side of the cut (e.g. cuts 2 and 3, or 5 and 6) due to symmetry. Values of stress correction in Fig. 10(a), at specific $x$ positions, appear in Fig. 9(c). The total stress (Fig. 9(d)) shows that the greatest residual stress occurs near the center of the plate, with nearly an identical residual stress profile for $3 W / 8<x<5 W / 8$ (cuts 5, 2, and 4). Residual stress decreases with distance from the sample center, with the decreases along $x$ and $y$ being symmetric about the sample center.

Figure 11 shows a color map of total measured longitudinal BRS $\left(\sigma_{x x}\right)$ in sample F8 as a function of in-plane position with dashed lines to represent the boundaries of CMOD measurements. The map shows that there is compressive BRS near the boundaries and tensile BRS near the center. Figure 12 shows a color map of total longitudinal BRS in sample G14 generated following the same procedures. Sample G14 is cut from a different bar (G rather than F) and is located closer to the end of the quenched parent stock than

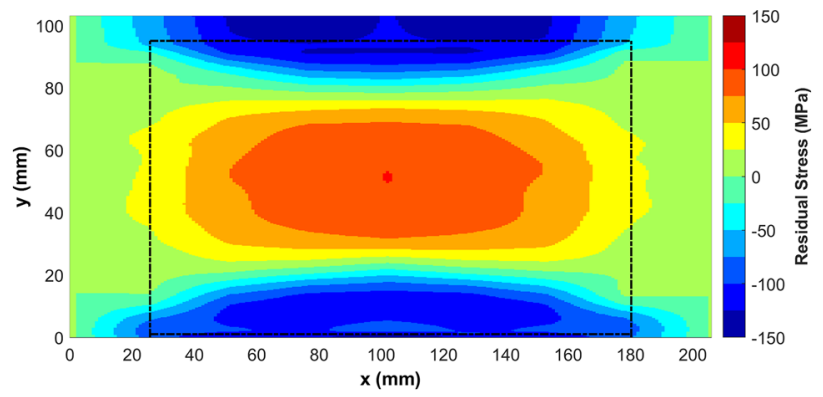

Fig. 11 Color map of the longitudinal bulk residual stress $\left(\sigma_{\mathrm{xx}}\right)$ in sample F8 where the dashed line represents the boundaries of CMOD measurements 


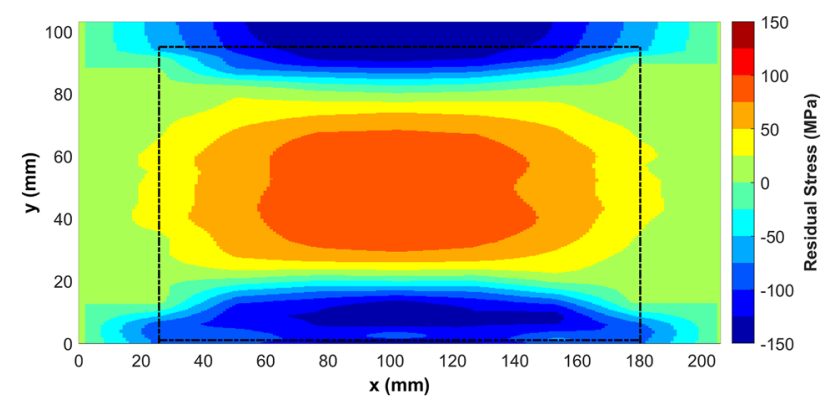

Fig. 12 Color map of the longitudinal bulk residual stress $\left(\sigma_{\mathrm{xx}}\right)$ in sample G14 where the dashed line represents the boundaries of CMOD measurements

sample F8. Despite the differences in bar and location in the parent stock, there is a clear similarity of longitudinal BRS in the two samples. This indicates that the quenching and sectioning processes produced individual samples with quite similar BRS fields. The similarity in the BRS field among these samples is further reinforced by assessing the pointwise average of the two longitudinal BRS measurements and the absolute values of differences between them, as shown in Figs. 13 and 14, respectively. The RMS difference over all locations is $8.4 \mathrm{MPa}\left(8.6 \%\right.$ of maximum $\left.\sigma_{\mathrm{xx}}\right)$. Figure 14 shows that the largest differences ( 15 to $30 \mathrm{MPa}$ ) occur near the start of the cuts, which may result from lower precision of slitting for short cut depths [17].

\section{Transverse BRS Mapping}

Figure 15 shows results of $\sigma_{y y}$ mapping measurements on sample F7 previously bisected at $x=H / 2$, as shown in Fig. 6, comprising CMOD versus cut depth, measured stress, stress corrections from prior cuts, and total stress. Compared to CMOD measured for cut $2 \mathrm{~L}$, there is a systematic decrease in the magnitude of measured CMOD for subsequent cuts (see Fig. 15(a)). This is consistent with the release of stress in prior measurements. CMOD data fall into pairs (Fig. 15(b)), with cuts at symmetric positions $3 \mathrm{~L}$ and $4 \mathrm{~L}$ being similar.

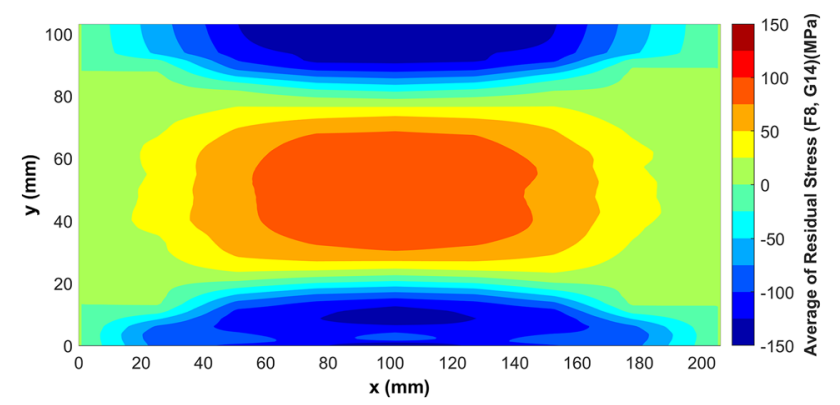

Fig. 13 Color map of the average of the longitudinal bulk residual stress $\left(\sigma_{\mathrm{xx}}\right)$ in samples F8 and G14

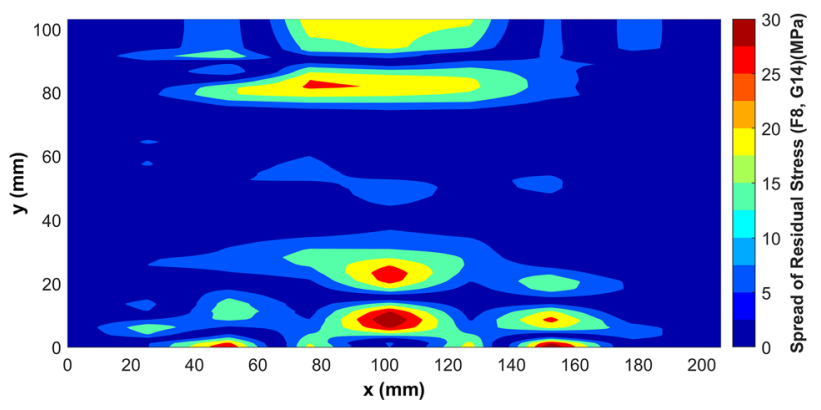

Fig. 14 Color map of the absolute values of difference of the longitudinal bulk residual stress $\left(\sigma_{\mathrm{xx}}\right)$ in samples F8 and G14

The stress corrections (Fig. 15(c)) show the effect of cut 2L being significant at cut locations $3 \mathrm{~L}$ and $4 \mathrm{~L}$ and the effect of cut 1 being significant at cut locations $2 \mathrm{~L}, 3 \mathrm{~L}$, and $4 \mathrm{~L}$ for $\mathrm{x}>80 \mathrm{~mm}$ and almost negligible at $x<80 \mathrm{~mm}$. To aid visualization, Fig. 10(b) shows the effect of $\sigma_{x x}$ at cut 1 on $\sigma_{y y}$ in sample F8 as a 2D map; results are similar for sample F7. On account of symmetry, corrections for cuts in the right half of the plate $(2 R, 3 R$, and $4 R$ ) are identical to those in the left half. The total stress in Fig. 15(d) is the sum of the measured stress and each applicable correction. The plot in
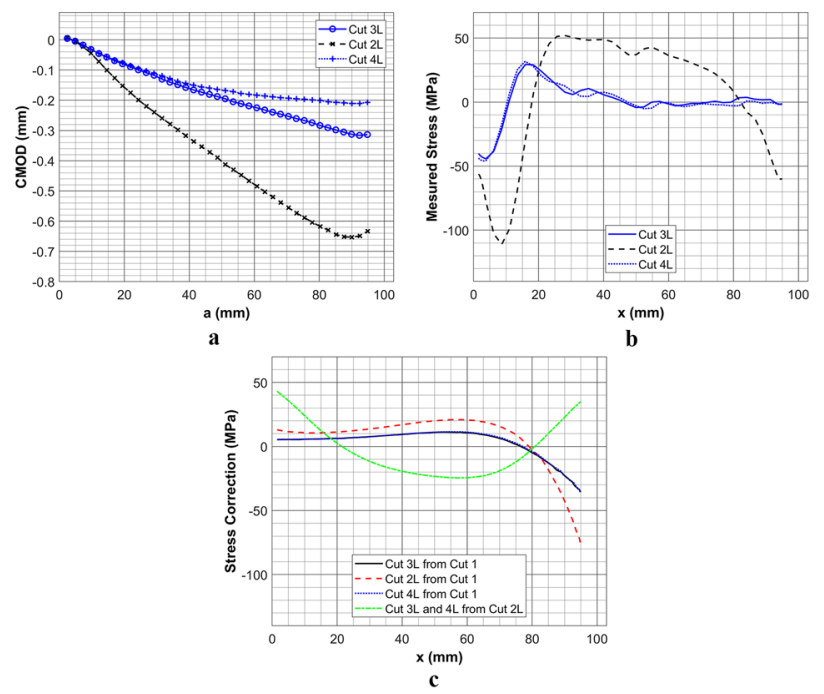

b
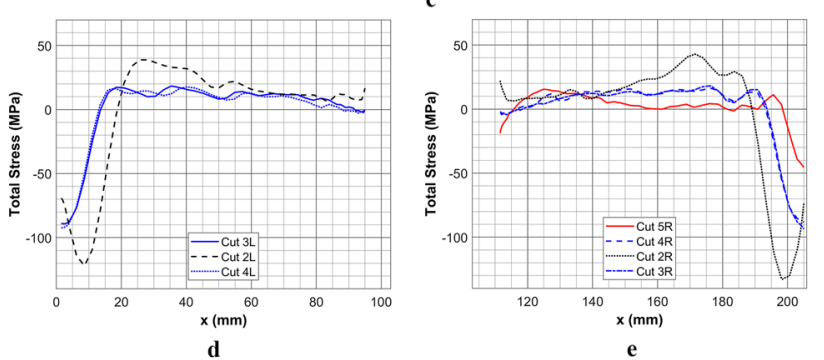

Fig. 15 Data for $\sigma_{\mathrm{yy}}$ measurements in sample F7, (a) CMOD versus cut depth, (b) measured stress (c) stress corrections and (d) total stress for the left half, and (e) total stress for the right half 


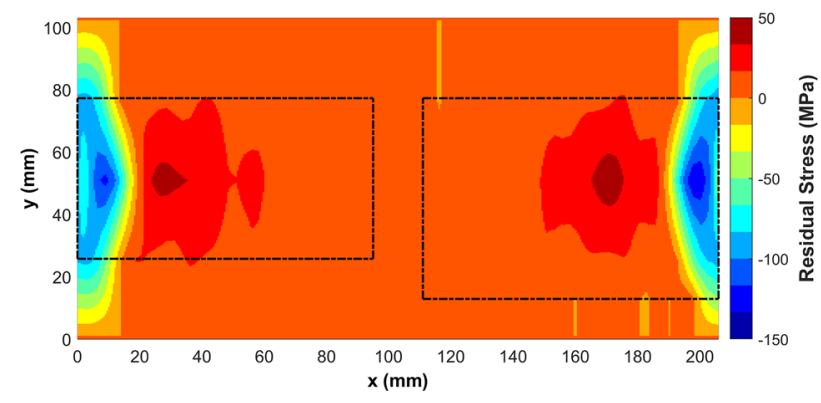

Fig. 16 Color map of total transverse bulk stress $\left(\sigma_{\mathrm{yy}}\right)$ in sample F7 where the dashed lines represent the boundaries of CMOD measurements

Fig. 15(e) includes a transformation of the depth positions for measurements in the right half of the sample, so that they run from $x=H(206 \mathrm{~mm})$ to $x=H / 2$, which allows Fig. 15(d), (e) to provide a full view of the $\sigma_{y y}$ field. Near the part edges $(x=0$ or $206 \mathrm{~mm}$ ) the residual stress is compressive, rising into low level tension (20-40 MPa) at $25 \mathrm{~mm}$ from the edges, and then monotonically decreasing toward near zero at the center of the sample. Figures 16 and 17 show color maps of the transverse BRS $\left(\sigma_{\mathrm{yy}}\right)$ in samples F7 and D1, respectively, with dashed lines to represent the boundaries of CMOD measurements. The color map in Fig. 18 shows the average of the transverse BRS in these two samples and Fig. 19 shows the absolute values of differences between them. The RMS difference overall points is $4.5 \mathrm{MPa}\left(12.1 \%\right.$ of maximum $\left.\sigma_{y y}\right)$. As was observed for the longitudinal BRS (Fig. 14) the greatest differences occur near the boundaries ( $x=0$ and $206 \mathrm{~mm}$ ). The $\sigma_{y y}$ field is symmetric about the sample center, along both $x$ and $y$, with the magnitude of $\sigma_{y y}$ being substantially lower than was found for $\sigma_{x x}$ (compare Figs. 13 and 18). The fact that $\sigma_{x x}$ is larger than $\sigma_{y y}$ is consistent with the sample size being larger along $x(206 \mathrm{~mm})$ than along $y(102 \mathrm{~mm})$.

Figure 20 shows results of a validation contour measurement of the longitudinal BRS in sample G7 at $x=H / 2$. The sample was not notched prior to measurement. The trend of BRS from contour is similar to the trend from CMOD slitting at $x=H / 2$ (Fig. 7)

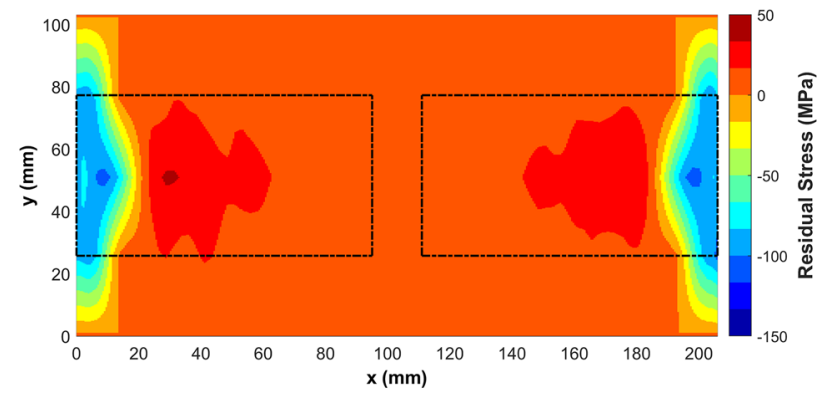

Fig. 17 Color map of total transverse bulk stress $\left(\sigma_{\mathrm{yy}}\right)$ in sample D1 where the dashed lines represent the boundaries of CMOD measurements

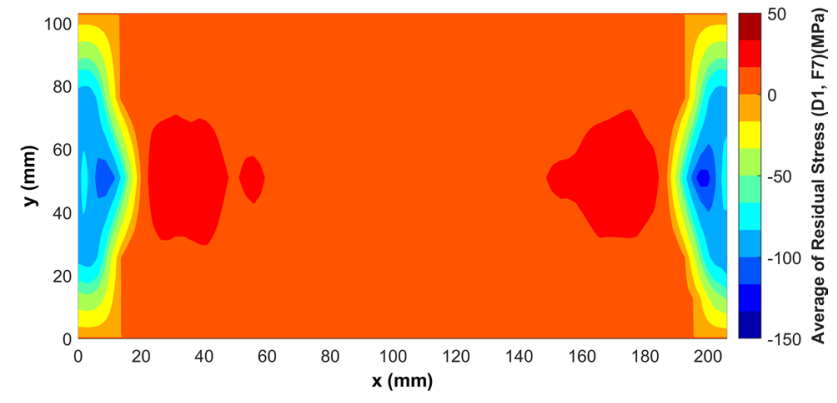

Fig. 18 Color map of the average of the transverse bulk residual stress $\left(\sigma_{\mathrm{yy}}\right)$ in samples D1 and F7

with high compressive stress (-180 MPa) near the boundaries and tensile stress (100 MPa) near the center. Figure 20 shows a slight variation of stress through the $28 \mathrm{~mm}$ thickness, particularly within $2 \mathrm{~mm}$ of the top and bottom surfaces.

Figure 21 shows results of a validation contour measurement of the transverse BRS in sample G8 at $y=W / 2$. There are regions of compressive stress near the left and right boundaries, adjacent areas of tensile stress, and low stresses near the center.

Figure 22 compares the thickness average of the contour results with CMOD slitting results. The trend in longitudinal BRS at $\mathrm{x}=H / 2$ (Fig. 22(a)) from CMOD slitting and contour are similar, with high compressive stress (-160 to $-120 \mathrm{MPa}$ ) at the boundaries and tensile stress (around $100 \mathrm{MPa}$ ) near the center. Figure 22(b) compares results for transverse BRS at $y=W / 2$ from CMOD slitting and contour. There is high compressive stress at the boundaries (-100 to -140 MPa), a tensile stress peak ( $40 \mathrm{MPa}$ ) adjacent to the compression, and a monotonic decrease to near zero stress at the center. Overall, there is excellent agreement between the BRS data from CMOD slitting and contour, except near the edges where there are differences of 10 to $40 \mathrm{MPa}$ for longitudinal BRS and a 10 to $20 \mathrm{MPa}$ for transverse BRS. RMS differences between the line profiles from contour and slitting are $14 \mathrm{MPa}$ and $8 \mathrm{MPa}$ for longitudinal and transverse BRS, respectively.

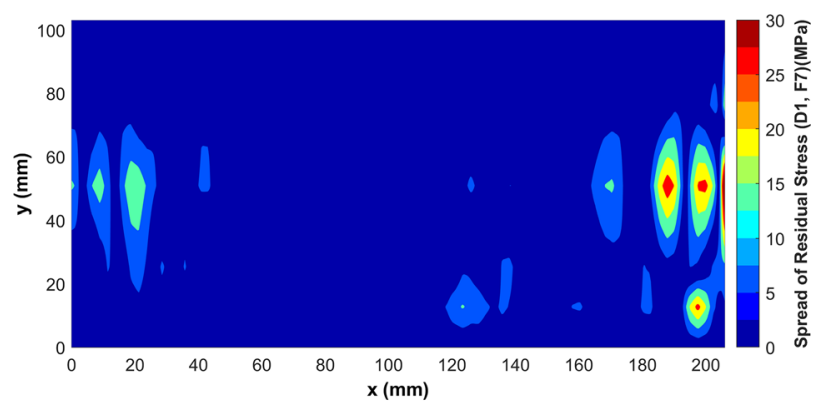

Fig. 19 Color map of the absolute values of difference of the transverse bulk residual stress $\left(\sigma_{\mathrm{yy}}\right)$ in samples D1 and F7 


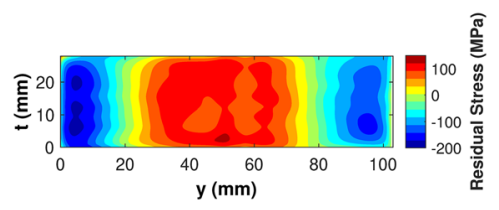

Fig. 20 Color map from a contour measurement of longitudinal BRS component $\left(\sigma_{\mathrm{xx}}\right)$ in sample $\mathrm{G} 7$

\section{Discussion}

\section{Level of Residual Stress}

The measured BRS in this work is consistent with prior work by Olson and Hill [9] and Prime and Hill [18] in quenched aluminum. The shape of the longitudinal $\left(\sigma_{x x}\right)$ stress field (Fig. 13) is roughly that of an elliptic paraboloid, which is consistent with [9] for slices cut from a smaller quenched bar. The magnitude of BRS from cut 1 in Fig. 9(d) is consistent with measurements of BRS in square plate-like samples in [18]. The consistency of results between this study and prior work shows that quenching produces similar stress fields in both shape and magnitude, despite differences in the size and shape of samples.

The high level of residual stress shown in Figs. 13 and 18 is atypical of service and highlights the utility of stress relief by stretching [18]. To show the difference, BFS slitting measurements were made in samples of the same dimension but cut from stress relieved block A (Fig. 1). Results show that longitudinal (Fig. 23(a)) and transverse (Fig. 23(b)) stress levels are far lower in the stress relieved condition. The stretch stress relief process provides material with stress $<20 \mathrm{MPa}$, which is about a factor of 8 less than for the quenched material.

\section{Comparison of CMOD and BFS Slitting}

This paper introduces the concept of using CMOD measurements for slitting to resolve a one-dimensional profile of residual stress. The results demonstrate that this approach provides results similar to those found from BFS slitting with some differences at shallow cut depths (Fig. 7(c)). To evaluate more fully the differences between CMOD and BFS

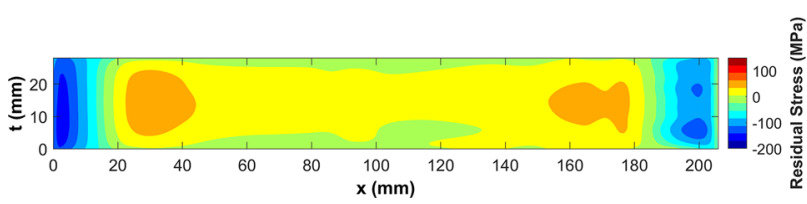

Fig. 21 Color map from a contour measurement of transverse BRS component $\left(\sigma_{\mathrm{yy}}\right)$ in sample G8
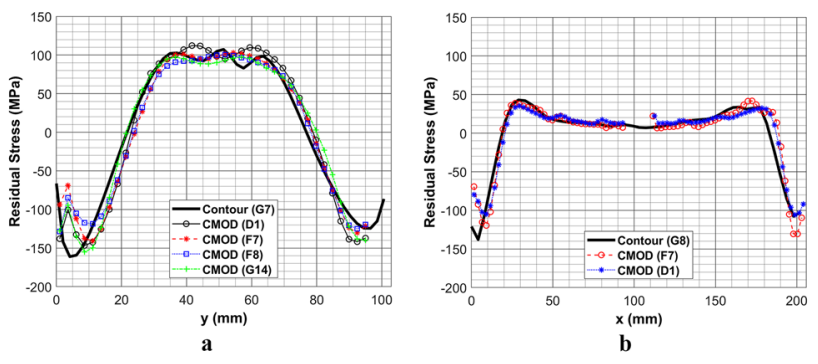

Fig. 22 Comparison of (a) measured longitudinal component $\left(\sigma_{\mathrm{xx}}\right)$ of BRS at $x=H / 2$ and (b) measured transverse component $\left(\sigma_{\mathrm{yy}}\right)$ of BRS at $y=W / 2$ using CMOD slitting and contour

slitting, it is useful to assess potential for uncertainty and observed variability.

To assess potential for uncertainty, we follow the prior work of Olson and Hill [9] which uses deformation versus cut depth responses for Legendre polynomial stress fields to compute inherent uncertainty. Finite element models using Legendre polynomial inputs, representing plausible equilibrium residual stress fields, are developed for a range of plate aspect ratio $(H / W)$. CMOD and BFS are determined from the model outputs as functions of slit depth and Legendre polynomial order (second or higher order). The uncertainty is computed using these deformations following the uncertainty estimation procedure developed by Prime and Hill [19]. Figure 24 shows deformation versus cut depth for various Legendre polynomial stress fields for aspect ratio $H / W=2$ (dimension along the slit depth half that perpendicular to the slit). The deformation versus cut depth trends for BFS and CMOD have very similar shape but opposite sign and differing magnitude. The shape similarity suggests that either measure of deformation offers similar utility for residual stress measurement. Figure 25 shows the RMS inherent uncertainty for BFS and CMOD slitting. The uncertainties in stress are proportional to the specific uncertainties in measurand assumed in the analysis, which were $1 \mu \varepsilon$ for BFS and $1 \mu \mathrm{m}\left(W / 10^{5}\right)$ for CMOD
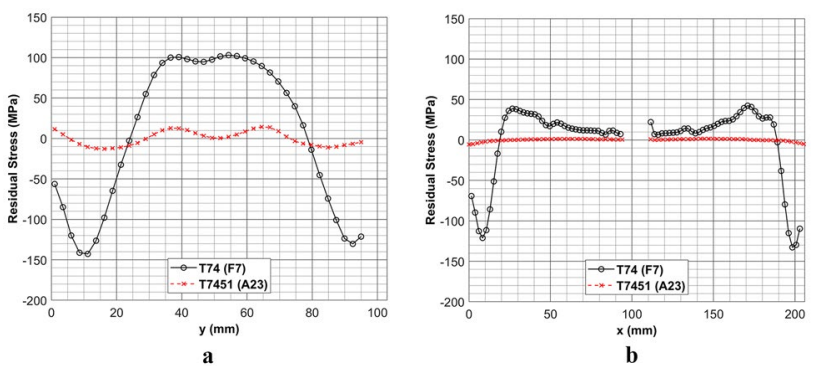

Fig. 23 Comparison of residual stress in sample F7 in T74 condition (measured with CMOD slitting) to residual stress in sample A23 in T7451 condition (measured with BFS slitting): (a) $\sigma_{\mathrm{xx}}$ at $x=H / 2$, and (b) $\sigma_{\text {yy }}$ at $y=W / 2$ 


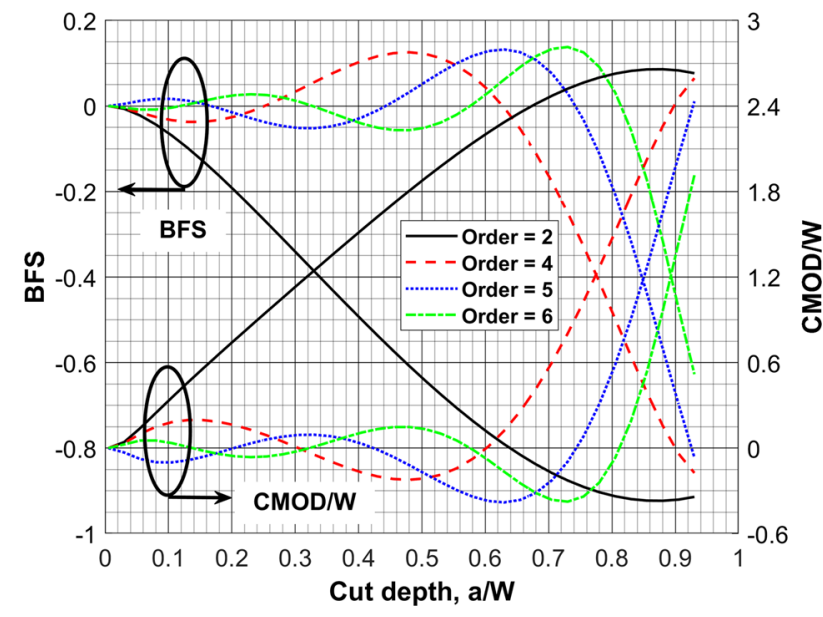

Fig. 24 Comparisons of deformation versus cut depth for $H / W=2$ for a range of Legendre polynomial order for $\mathrm{E}=1$ for $\mathrm{CMOD}$ $\left(L_{\mathrm{M}} / W=0.0625\right)$ and $\operatorname{BFS}\left(L_{\varepsilon} / W=0.0156\right)$

(the readout resolutions of our instrumentation). For BFS slitting, uncertainty increases as $H / W$ decreases, which was noted earlier in [9] and limits BFS slitting to aspect ratios $H / W>0.4$. On the contrary, the results for CMOD slitting show that it becomes more precise for decreasing $H / W$. Figure 26 compares CMOD (Fig. 26(a)) and BFS (Fig. 26(b)) deformations for a uniform stress field and a range of $H / W$. In general, larger deformations and steeper slopes indicate greater potential fidelity. For decreasing $H / W$, CMOD increases while BFS decreases, consistent with inherent uncertainty of Fig. 25. For $H / W=0.50$, there is a discontinuity in BFS at $a / W=0.25$ because the sign of BFS reverses

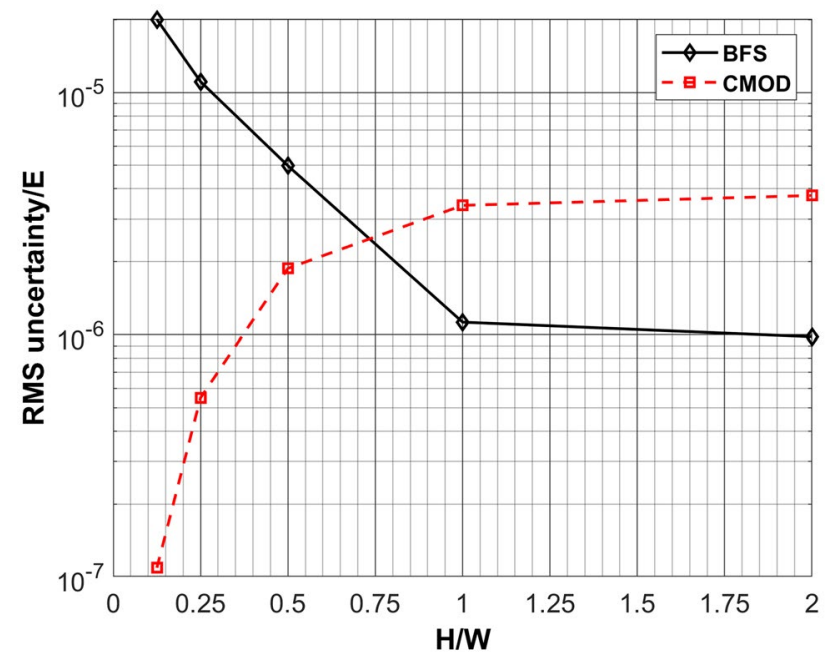

Fig. 25 Comparisons of uncertainty in stress normalized by elastic modulus as a function of plate aspect ratio $(H / W)$ for CMOD $\left(L_{\mathrm{M}} / W=0.0625\right.$, precision of $\left.W / 10^{5}\right)$ and BFS $\left(L_{\varepsilon} / W=0.0156\right.$, precision of $1 \mu \varepsilon$ )

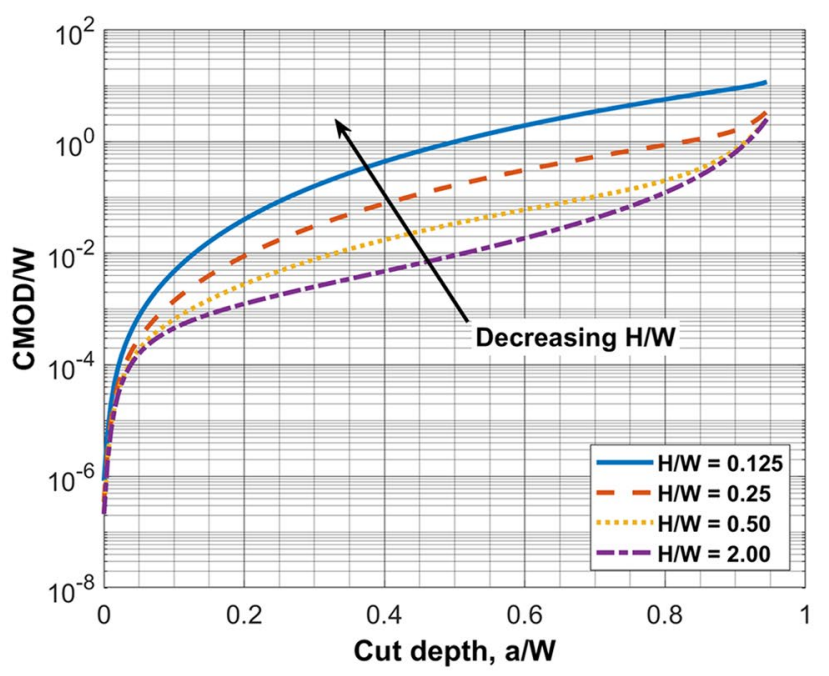

a

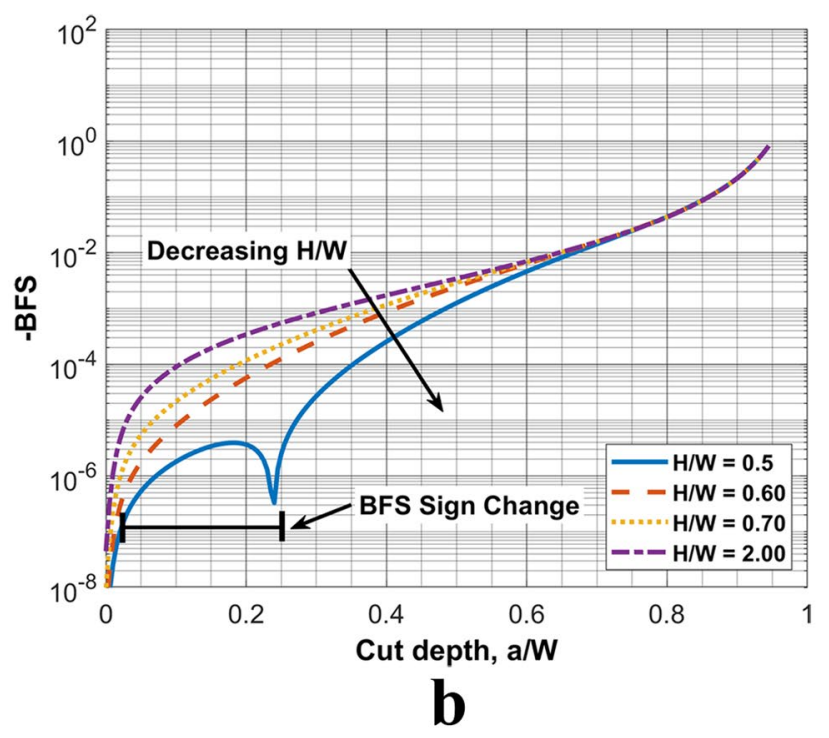

Fig. 26 Comparison of deformation versus cut depth for uniform stress along the cut plane $(\sigma / \mathrm{E}=0.001)$ and different aspect ratios $(H / W)$ for (a) $\operatorname{CMOD}\left(L_{\mathrm{M}} / W=0.0625\right)$ and (b) BFS $\left(\mathrm{L}_{\varepsilon} / \mathrm{W}=0.0156\right)$; for $H / W=0.5$, BFS reverses sign at $a / W=0.25$

(-BFS is $<0$ for $a / W<0.25$ ). This change of sign leads to poor conditioning of BFS slitting for small $H / W$.

Repeatability standard deviation has been assessed in prior studies of BFS slitting and can be compared to the present results for CMOD slitting. Lee and Hill [8] assessed repeatability in six replicate samples that had been laser shock peened. They found repeatability standard deviation between 5 and $10 \mathrm{MPa}$ for samples with $H / W>2$. Olson and Hill [9] performed repeated BFS slitting measurements in quenched aluminum plates with $H / W=1.5$. They found repeatability standard deviation ranging from 3 to $7 \mathrm{MPa}$. In the present work, among the four CMOD slitting 
measurements at $x=H / 2$ with $H / W=2$ (Fig. 22(a)), we find the repeatability standard deviation ranging from 3 to $16 \mathrm{MPa}$, which suggests that CMOD slitting has repeatability comparable to that found earlier for BFS slitting at similar $H / W$.

\section{Notch Correction Significance}

The notch cut to accommodate the CMOD gage is expected to have a small but measurable effect on measured stress. Figure 3(d) shows that the magnitude of the effect is about $80 \%$ at the notched edge, falls rapidly to $10 \%$ at $y / W=0.05$, and reaches less than $2 \%$ for $y / W>0.10$. Figure 8 shows a correction intended to account for the notch effect, which reinforces the notion that the effect is highly localized. Overall, the correction does not appear to significantly improve the stress profile. The consequence of the notch effect depends on the use of the data; for this study, aimed at revealing the stress profile across the part, the localized effect is of minor consequence. Because the size and scale of the notch effect depend on the notch dimensions ( $L_{\mathrm{M}}$ and $D_{\mathrm{M}}$ in Fig. 2), it may be useful in other experiments to use a CMOD gage (or other sensor for measuring CMOD) that would not disturb the preexisting stress field.

\section{Conclusion}

This study has introduced the concept of using CMOD for slitting residual stress measurements, validated it against typical BFS slitting, and applied it to map two components of residual stress in quenched aluminum plate-shaped samples. A direct comparison of residual stress determined from slitting using CMOD and BFS measurements shows that the techniques give comparable results to within $25 \mathrm{MPa}$ maximum pointwise difference and 7.2 MPa RMS difference. CMOD slitting was used to construct two-dimensional maps of residual stress in AA7050-T74 plate-shaped samples. The resulting residual stress maps were similar to those found in recent studies of residual stress in quenched aluminum, with stress along the length being -150 MPa at the exterior and $100 \mathrm{MPa}$ at the interior, and stress across the width being $-120 \mathrm{MPa}$ at the exterior and $20 \mathrm{MPa}$ at the interior. Measurements on replicate parts exhibited RMS differences of 8.4 $\mathrm{MPa}$ and maximum difference of $38 \mathrm{MPa}$. CMOD slitting was found to work well for a wide range of plate aspect ratio, offering advantage over BFS slitting, which is limited to relatively wide plates. Overall, CMOD slitting is a useful complement to BFS slitting that provides similar quality of results while using a gage with a simpler mechanical attachment and being applicable to a wider range of geometry (and in particular to narrow samples).

Acknowledgements This work was performed within a collaborative project between the University of California, Davis (UC Davis) and the Technische Universität Kaiserslautern (TUK). We acknowledge discussions with collaborators at UC Davis (Christopher D'Elia, Barbara Linke) and at TUK (Daniel Weber, Benjamin Kirsch, Jan Aurich). UC Davis received funding from the National Science Foundation under Award No. 1663341 (Division of Civil, Mechanical and Manufacturing Innovation (CMMI)). Any opinions, findings, and conclusions or recommendations expressed in this material are those of the authors and do not necessarily reflect the views of the National Science Foundation. TUK received financial support from Deutsche Forschungsgemeinshaft (DFG) within project AU 185/64-1.

\section{Declarations}

Conflict of Interest The authors have no conflicts of interest to disclose.

Open Access This article is licensed under a Creative Commons Attribution 4.0 International License, which permits use, sharing, adaptation, distribution and reproduction in any medium or format, as long as you give appropriate credit to the original author(s) and the source, provide a link to the Creative Commons licence, and indicate if changes were made. The images or other third party material in this article are included in the article's Creative Commons licence, unless indicated otherwise in a credit line to the material. If material is not included in the article's Creative Commons licence and your intended use is not permitted by statutory regulation or exceeds the permitted use, you will need to obtain permission directly from the copyright holder. To view a copy of this licence, visit http://creativecommons.org/licenses/by/4.0/.

\section{References}

1. Totten GE, Mackenzie DS (2000) Aluminum Quenching Technology: A Review. Materials Science Forum - Mater Sci Forum 331-337:589-594. https://doi.org/10.4028/www.scientific.net/MSF. 331-337.589

2. Masoudi S, Saeid A, Ehsan S, Hamdollah E (2015) Effect of machining-induced residual stress on the distortion of thin-walled parts. Int J Adv Manuf Tech 76(1):597-608. https://doi.org/10.1007/ s00170-014-6281-x

3. Zhang S, Wu Y, Gong H (2012). A modeling of residual stress in stretched aluminum alloy plate. J Mater Process Technol. 212: 2463-2473. https://doi.org/10.1016/j.jmatprotec.2012.06.019

4. Schajer GS, Ruud CO (2013) Overview of Residual Stresses and Their Measurement. In: Schajer GS (ed) Practical Residual Stress Measurement Methods. Wiley, United Kingdom, pp 1-27. https:// doi.org/10.1002/9781118402832.ch1

5. Hill MR (2013) The Slitting Method. In: Schajer GS (ed) Practical Residual Stress Measurement Methods. Wiley, United Kingdom, pp 89-106. https://doi.org/10.1002/9781118402832.ch4

6. Cheng W, Finnie I (2007) Measurement of Through-Thickness Residual Stress in Residual Stress Measurement and the Slitting Method, Ch. 7, pp 103-116, Springer Science + Business Media, LLC., New York. https://doi.org/10.1007/978-0-387-39030-7

7. Schajer G, Prime M (2006) Use of Inverse Solutions for Residual Stress Measurement. J Eng Mater Technol 128:375-382. https://doi. org/10.1115/1.2204952 
8. Lee MJ, Hill MR (2007) Intralaboratory repeatability of residual stress determined by the slitting method. Exp Mech 47(6):745-752. https://doi.org/10.1007/s11340-007-9085-1

9. Olson MD, Hill MR (2018) Two-Dimensional Mapping of In-Plane Residual Stress with Slitting. Exp Mech 58:151. https://doi.org/10. 1007/s11340-017-0330-y

10. Wong W, Hill MR (2013) Superposition and Destructive Residual Stress Measurements. Exp Mech 53:339-344. https://doi.org/10. 1007/s11340-012-9636-y

11. Pagliaro P, Prime MB et al (2011) Measuring Inaccessible Residual Stresses Using Multiple Methods and Superposition. Exp Mech 51:1123-1134. https://doi.org/10.1007/s11340-010-9424-5

12. ASTM E1820 (2017), Standard Test Method for Measurement of Fracture Toughness, ASTM International, West Conshohocken, PA. https://www.astm.org/Standards/E1820

13. Hill MR, Olson MD, DeWald AT (2018) Repeatability of Contour Method Residual Stress Measurements for a Range of Materials, Processes, and Geometries. Materials Performance and Characterization 7(4):427-445. https://doi.org/10.1520/MPC20170044

14. Handbook ASM (1991) Heat Treating of Aluminum Alloys. ASM Handbook 4:841-879. https://doi.org/10.1361/asmhba0001205
15. Lee MJ, Hill MR (2007). Effect of strain gage length when determining residual stress by slitting, J Eng Mater Technol 129(1):143-150. https://doi.org/10.1115/1.2400263 See also an important erratum (2009) 131(4):047001. https://doi.org/10.1115/1.3227670

16. Prime MB, DeWald AT (2013) The Contour Method. In: Schajer GS (ed) Practical Residual Stress Measurement Methods. Wiley, United Kingdom, pp 109-138. https://doi.org/10.1002/9781118402832.ch5

17. Olson MD, DeWald AT, Hill MR (2020) An Uncertainty Estimator for Slitting Method Residual Stress Measurements Including Influence of Regularization. Exp Mech 60:65-79. https://doi.org/ 10.1007/s11340-019-00535-x

18. Prime MB, Hill MR (2002) Residual Stress, Stress Relief, and Inhomogeneity in Aluminum Plate. Scripta Mater 46(1):77-82. https:// doi.org/10.1016/S1359-6462(01)01201-5

19. Prime MB, Hill MR (2006) Uncertainty, Model Error, and Order Selection for Series-Expanded, Residual-Stress Inverse Solutions. J Eng Mater Technol 128(2):175-185. https://doi.org/10.1115/1. 2172278

Publisher's Note Springer Nature remains neutral with regard to jurisdictional claims in published maps and institutional affiliations. 